\title{
A qualitative study of consumer perceptions and use of traffic light food labelling in Ecuador
}

\author{
Wilma B Freire, William F Waters*, Gabriela Rivas-Mariño, Tien Nguyen and \\ Patricio Rivas \\ Institute for Research in Health and Nutrition, Universidad San Francisco de Quito, Vía Interoceánica s/n, \\ Circulo de Cumbayá, Quito, Ecuador
}

Submitted 20 January 2016: Final revision received 26 July 2016: Accepted 1 August 2016: First published online 13 September 2016

\begin{abstract}
Objective: To analyse patterns of knowledge, comprehension, attitudes and practices regarding the traffic light label placed on processed food packages to inform Ecuadorian consumers about levels of added fat, sugar and salt.

Design: Twenty-one focus group discussions organized by age group, sex and place of residence. Interviews with representatives of companies that manufacture or market processed foods. Analysis of regulations and structured observations of processed food labels.

Setting: Cities and towns in Ecuador's coastal, highland and eastern lowland regions. Subjects: One hundred and seventy-eight participants in twenty-one focus group discussions and nine key informants.

Results: Focus group participants knew about the traffic light label and understood the information it conveys, but not all changed their attitudes and practices related to the purchase and consumption of processed foods. Children, adolescents and adult males reported using the information infrequently; adolescents interested in health and adult women used the label the most to select products. Representatives of companies that manufacture or market processed foods generally opposed the policy, stating that the information is misleading. Nevertheless, some companies have reduced levels of added fat, sugar or salt in their products.

Conclusions: The traffic light label is an effective tool for conveying complex information. Its potential contribution to reduce consumption of products with high levels of fat, sugar and salt could be enhanced by promoting healthy diets among consumers who have not changed purchasing and consumption behaviour, by placing the label on front panels and by monitoring the production and marketing of processed foods.
\end{abstract}

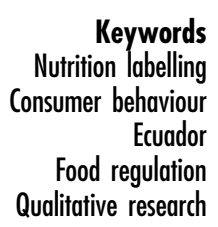

According to Ecuador's 2012 national health and nutrition survey (ENSANUT-ECU), overweight and obesity together affect a total of $8.6 \%$ of children under the age of 5 years, $29.9 \%$ of children aged $5-11$ years, $26.0 \%$ of adolescents aged $12-19$ years and $62 \cdot 8 \%$ of adults ${ }^{(1)}$. These figures mirror the global overweight and obesity epidemic that is associated with the increasing prevalence of chronic diseases, including diabetes, which is the leading cause of death in Ecuador ${ }^{(2,3)}$

Although the WHO Director-General has observed that no country has reversed the overweight and obesity epidemic in all age groups ${ }^{(4)}$, many countries have adopted policies having that goal, including the required use of visible and easily understood nutritional labels. Evidence from the USA, New Zealand, Australia ${ }^{(5)}$, the $\mathrm{UK}^{(6)}$ and other European countries ${ }^{(7-9)}$, as well as Asia, Africa, the
Middle East and Latin America ${ }^{(10)}$, suggests that nutritional labels can help consumers make healthy decisions regarding the purchase and consumption of foods, which in turn could contribute to preventing overweight and obesity $^{(11-14)}$. Evidence also suggests that consumers want easily understood nutrition information and research has shown that colourful front-of-package graphic displays respond most effectively to consumers' expectations in this regard ${ }^{(15-17)}$. In particular, traffic light (TL) labels provide visible and easily understood information on the nutritional content of food products ${ }^{(5,16,17)}$.

After reviewing policies implemented in other countries, the Ecuadorian Government promulgated a regulation in August 2014 which required that packages of processed foods carry TL labels with horizontal red, yellow and green bars placed in that order from top to bottom, as shown in 
Fig. 1. The regulation defines processed foods as packaged and branded products that have been submitted to technological processes for their transformation, modification or conservation ${ }^{(18)}$; they include beverages sweetened with sugar, but not products like milk, natural juices, grains, vegetables, fruit or meat that are packaged without transformation. The regulation stipulates that labels must be framed and placed on a white or grey background and must be proportional in size to the packages' principal panel; the label need not be placed on the front of the package, however. Text located on each bar indicates high, medium or low levels of fat, sugar or salt present in processed foods.

Cut-off points for high, medium or low concentrations of the three key ingredients were based on the British system $^{(19,20)}$, but were modified to raise the cut-off point for fat and to lower those for sugar and salt in order to reflect Ecuadorian consumption patterns revealed in the national survey $^{(1)}$. Table 1 shows the cut-off points for total fat, sugar and salt as established in the Ecuadorian regulation.

No previous research has been conducted in Ecuador to evaluate consumers' knowledge, understanding, attitudes and practices related to the purchase and consumption of

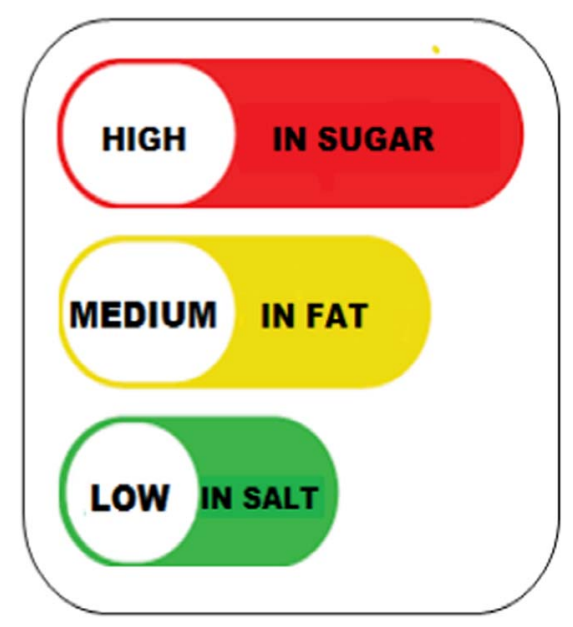

Fig. 1 (colour online) The Ecuadorian traffic light food label, translated from Spanish to English. The order of presentation of the horizontal bars is red, yellow and green, corresponding to high, medium and low concentrations of sugar, fat and salt. The yellow and green bars are proportionately shorter than the red bars processed foods. The current paper presents the results of a qualitative research project carried out in 2015 (one year after implementation of the policy) to evaluate the perceptions of Ecuadorian children, adolescents and adults, as well as representatives of companies that produce or market processed foods.

\section{Methods}

The study was approved by the Institutional Review Board of the Universidad San Francisco de Quito. Data were collected in twenty-one focus group discussions (FGD) that included a total of 178 participants. FGD were organized by sex; age group (5-9, 10-14, 15-19 and 20-64 years); and residence in large, medium or small cities in five of Ecuador's twenty-four provinces in the three mainland regions (coast, highlands and eastern lowlands). The purpose of this distribution was to observe potential differences in perceptions among males and females; children in two age groups, adolescents and adults; socioeconomic differences in rural and urban places; and potential cultural variation among residents of Ecuador's three mainland regions. In addition, nine key informant interviews (KII) were conducted with representatives of large, medium and small firms that produce or market processed foods. Finally, through structured observations, relevant legislation and regulations were reviewed and labels on processed food packages were analysed ${ }^{(21-24)}$.

The FGD guide included ten questions designed to explore research questions related to participants' knowledge, comprehension, attitudes and practices related to food and the TL label ${ }^{(24)}$. KII focused on perceptions, attitudes and practices related to consumer preferences and changes in corporate strategies in response to consumer preferences. Two semi-structured questions were designed to elicit extensive responses from interviewees. Table 2 provides the questions used in FGD and KII and the dimensions they addressed. The full question guide, including follow-up questions that were asked when the respective points were not spontaneously mentioned by participants, is provided as the online supplementary material.

FGD were organized by local counterparts in each region and were conducted in public facilities such as schools that were free of noise and interruptions. Discussions lasted between 1 and $2 \mathrm{~h}$. Participants were not

Table 1 Cut-off points for total fat, sugar and salt in the Ecuadorian traffic light food label

\begin{tabular}{llll}
\hline & Solid or liquid & Low concentration & Medium concentration \\
\hline Total fat & Solid & $\leq 3 \mathrm{~g}$ per $100 \mathrm{~g}$ & $>3$ and $<20 \mathrm{~g} \mathrm{per} 100 \mathrm{~g}$ \\
& Liquid & $\leq 1.5 \mathrm{~g}$ per $100 \mathrm{ml}$ & $>1.5$ and $<10 \mathrm{~g} \mathrm{per} 100 \mathrm{ml}$ \\
Sugar & Solid & $\leq 5 \mathrm{~g}$ per $100 \mathrm{~g}$ & $>5$ and $<15 \mathrm{~g} \mathrm{per} 100 \mathrm{~g}$ \\
& Liquid & $\leq 2.5 \mathrm{~g}$ per $100 \mathrm{ml}$ per $100 \mathrm{~g}$ & $>2.5$ and $<7.5 \mathrm{~g} \mathrm{per} 100 \mathrm{ml}$ \\
Salt (sodium) & Solid & $\leq 120 \mathrm{mg}$ per $100 \mathrm{~g}$ & $>120$ and $<600 \mathrm{mg} \mathrm{per} 100 \mathrm{~g}$ \\
& Liquid & $\leq 120 \mathrm{mg}$ per $100 \mathrm{ml}$ & $>120$ and $<600 \mathrm{mg} \mathrm{per} 100 \mathrm{ml} \quad \geq 7.5 \mathrm{~g} \mathrm{per} 100 \mathrm{ml} \quad \geq 600 \mathrm{mg} \mathrm{per} 100 \mathrm{~g}$ \\
\hline
\end{tabular}


Table 2 Focus group discussion and key informant interview questions and dimensions

A. Focus group discussions

1. The first question is about the foods that you eat or buy. When you buy these foods, how do you decide what to buy or eat? Comprehension, practices

2. These days, people talk a lot about nutrition and healthy foods. Could you tell me what these terms mean to you and what you know about them? Knowledge, attitudes

3. Have you heard or do you know about processed foods? What do you know about them? Knowledge

4. Now that we have discussed nutrition, healthy foods, unhealthy foods and processed foods, can you explain why these ideas are important to you when you buy or consume foods? Attitudes, practices

5. Now we are going to talk about the label that is called the traffic light. The traffic light on packages of processed foods was instituted in Ecuador in 2014. Could you please tell me what you have seen or heard about it? (Includes exercise with cards that have different versions of the traffic light label.) Attitudes, practices

6. I'm going to show you a card with a different label. Could you tell me what you think of it? (Exercise with cards with an example of the GDA label.) Knowledge, attitudes

7. Now l'd like to hear your recommendations about promoting healthy consumption. Comprehension, attitudes

8. What other messages have you seen on food packages? Knowledge

9. Do you think it is important to include any other information on the packages? Attitudes

10. Is there any other point about the traffic light label that you would like to talk about? Comprehension, attitudes

B. Key informant interviews

1. What are the perceptions of your company regarding the traffic light label? Attitudes, practices

2. What are the perceptions with regard to the preferences and behaviour of your customers and the public in general? Attitudes

GDA, Guideline Daily Amount.

compensated but were served healthy refreshments. FGD were recorded and transcribed. KII were conducted in corporate offices and extensive notes were taken. Transcriptions were analysed using a three-stage coding process based on the grounded theory approach. First, open coding identified basic concepts and labels through a line-by-line microanalysis that led to a classification of categories and sub-categories and their underlying dimensions. Second, axial coding related underlying categories to their respective sub-categories, grouped them to dimensions and properties, and identified conditions under which those dimensions and properties were expressed. Third, selective coding integrated and refined key dimensions and the relationships between them in order to arrive at key themes that were central to the research ${ }^{(24)}$.

\section{Results}

This section reports on the knowledge, comprehension, attitudes and practices of FGD and KII participants with regard to the TL label and food purchase and consumption, as well as the results from analysis of regulations and structured observations of processed food labels.

\section{Focus group discussions}

\section{Knowledge}

When presented with cards that showed different versions of the TL label, nearly all FGD participants responded that they were familiar with it and stated that they had observed it on packages of processed foods. As one 34-year old woman responded, 'It's very common; even the bread that I buy has the traffic light food label.' Among the few participants who did not recognize the label were some children aged 5-9 years and several older adults. In contrast, children aged 10-14 years were very familiar with the label and mentioned it spontaneously when discussing snacks and sweetened beverages:

'They put the traffic light food label because here in Ecuador we have not been shown how to eat, so we have to begin to educate ourselves because we have begun to see a lot of obesity, and a lot of us are also dying.' (Female, 16 years)

\section{Comprebension}

Participants identified the TL label as a source of information on two different levels. First, nearly all stated that the information contained in the TL label could be useful on an individual level as a tool for decision making in food purchase and consumption. Because of its graphical nature, participants (even children) recognized that the label provides information on the content of fat, sugar and salt, although fat and sugar were mentioned more often than salt:

'The label is to see if there is fat and sugar in order to eat healthy, and whatever is not healthy, to avoid it. This is because some of these products have a lot of fat and sugar.' (Female, 8 years)

'The traffic light nutrition label is so that we understand whether something has sugar or not, like juices.' (Female, 9 years)

In particular, participants understood that the label informs consumers about the levels of salt, sugar and fat in different products and enables them to evaluate their contents in the context of their personal needs and preferences:

'The traffic light was put there so that people wouldn't buy foods that have a lot of fat, in order to have good nutrition.' (Male, 12 years)

'We don't all have knowledge or we don't have time to be looking when products come out: how many calories, how much fat. You know, it's in plain sight, where we can see.' (Male, 22 years) 
Second, several participants stated that a generalized understanding of the information might contribute to reducing obesity, diabetes and chronic diseases that they recognized as important problems in the Ecuadorian population:

'[The label] is so that we don't get sick. That's what I have seen in the news on TV; that one of the most prevalent illnesses here is diabetes. Most people have diabetes and the traffic light label is a campaign to inform the people, because a lot of people die of diabetes.' (Male, 14 years)

According to the FGD participants, TL labels are easily understood because of the bright colours and because of the similarity to everyday experience with traffic lights found in streets throughout the country:

'It's just like a traffic light when you're driving, right? You stop at the red, use caution with the yellow, and with the green, you go right ahead.' (Female, 16 years)

FDG participants accorded special importance to the red bars, associating them with warnings of potential danger. They understood that red bars do not necessarily mean that specific products should not be consumed, but rather that their purchase and consumption should be carefully considered. As one participant stated:

'When there is red, it's high in fat or salt; that is, we shouldn't eat it or at least we should eat less. We should be cautious; maybe we could eat a little, like in the case of processed meats. I don't think that anyone will stop consuming, but maybe not so frequently, or once in a while.' (Female, 39 years)

Participants often mentioned that foods that are not high in fat, sugar or salt are acceptable or healthy and that the TL label helps them to identify healthy alternatives:

'Some products just say medium, medium, low. This means that it is a product that is moderate for consumption.' (Female, 30 years)

While the label itself is well understood, many participants wanted to know more about the health effects of consuming fat, sugar and salt:

'The label is] easy to understand textually, but you can see that one should know a little more, to know the consequences of these elevated concentrations. You know, if the level of salt is low, if it has fat or it doesn't have sugar; and on the other hand, if another product is high in salt, medium in sugar, medium in fat: how you compare them.' (Male, 27 years)

Participants reported that they had seen the label on the back of packages, which they thought inhibits easy observation:

'It's all strategic; that's why they put the label on the back, because we grab a package and forget to look at the traffic light nutrition label.' (Female, 41 years)
'Some packages are pretty small and sometimes the traffic light label is on the back and it's small so people don't see it. Then they eat the product and ignore the traffic light.' (Male, 27 years)

With regard to its size, FGD participants stated that compared with lists of ingredients, the text on TL labels is large enough to be easily read:

'The text that reads high, medium or low is in capital letters. That's good because it can be read more easily.' (Female, 17 years)

When comparing cards with examples of the TL and Guideline Daily Amount (GDA) labels, participants preferred the former to the latter, which has been promoted by the food industry ${ }^{(21)}$ :

'The traffic light label is more recommendable because people aren't going to see other things. If I am in a hurry, I'm going to eat quickly. I'm not going to be perusing the percentages. On the other hand, the traffic light is more practical, to be able to follow [the information].' (Male, 17 years)

'The traffic light label is easier to understand. With this one [the GDA] we have to see the grams: $21 \%$. But the traffic light label already has the details on everything: whether it is high, medium, or low.' (Male, 34 years)

The TL label was also preferred over the black and white GDA label because of the striking colours:

'I'm shocked because they [the GDA labels] don't have the bright colours that the traffic light label does, which attracts more attention. These are very dark; they don't attract our attention.' (Female, 25 years)

'The traffic light label has stronger, pastel colors. When you form mental images, the red is like a warning to stop.' (Female, 20 years)

Children aged 10-14 years in particular reported that they have seen GDA labels on sweetened beverages. While it appears to provide more detailed information, it is not understood or used because of the way it is presented:

'Yes, this [GDA] label is on the beverage. Yes, there is; I think there are four of these rectangles, but not in colours, but rather black and white. But sometimes we ignore this and drink the beverage.' (Male, 34 years)

'They put it in black and white so you won't realize, and you buy more.' (Female, 28 years)

\section{Attitudes and practices}

Some FGD participants reported that they formulate attitudes about processed foods based on the information on 
the TL label. Nevertheless, these attitudes do not necessarily translate into changes in behaviour related to the purchase and consumption of processed foods. For example, participants reported that when they see packages with red labels, they do not necessarily change their decision regarding purchase and consumption. Rather, other factors (especially flavour) may prevail:

'It's very easy to understand the traffic light label, but it's hard to apply it. That is, we understand but we don't take it to heart day to day. It's there and I'm going to buy it because I like it.' (Female, 25 years)

Brand name recognition is also important to consumers, so that nutritional information may be less important in the selection of specific products, especially given the effect of advertising on preferences:

'Sometimes the publicity for some products is so extensive. For example, many times you are not going to buy just any yoghurt. Instead, you buy what you like, the one with the brand name, which you always see in television advertising. That's what attracts people the most.' (Female, 40 years)

Age is an important determinant of attitudes related to processed foods. In particular, the responses of children aged 5-9 and 10-14 years stand out. These age groups are critical because it has been suggested that children are particularly susceptible to television advertising of processed foods ${ }^{(25,26)}$. FGD results suggest a more nuanced pattern, however. The younger children demonstrated little knowledge of brands and limited understanding of the TL label because they do not purchase food on their own and have limited ability to comprehend written information. Thus, they seem relatively immune to advertising, expressing preferences for food prepared at home. In contrast, children 10-14 years of age are familiar with brand names of packaged snacks and sweetened beverages. Having been exposed to mass media advertising, they reported favourable attitudes related to processed foods that contain high concentrations of fat, sugar or salt, even though they are familiar with the TL label. They also enjoy greater autonomy because parents often give them small amounts of money to buy snacks from street vendors, neighbourhood shops or school cafeterias (which sometimes continue to sell processed foods despite regulations to the contrary). Hence, they make some independent decisions about food, even though adult FGD participants thought that their children pay little attention to nutritional information. As one female participant commented:

'Generally, the children don't look [at nutritional labels]. The mother is the one who buys food; she's the one who looks, but the children just grab the food, open it, and eat it. They don't look at those things; they're small.' (Female, 29 years)
Another factor that affects children's decisions about purchasing snacks is cost. Since they are given only small amounts of money, they prefer inexpensive items, including small packets of sweets, which do not always carry nutritional labels even though they have high concentrations of sugar.

In contrast, adolescents aged 15-19 years expressed knowledge and understanding of the TL label, but they do not necessarily change their behaviour because flavour and a sense of well-being that food conveys are more important. In fact, they may prefer products with high concentrations of sugar and salt. While some members of this age group, particularly university students, demonstrated some interest in health and nutrition, they feel that they have few alternatives to unhealthy but readily accessible products. Some participants in this age group also felt that nutritional information is not relevant to them because it applies only to the ill or elderly:

'I haven't noticed any difference in what I buy since the traffic light nutrition label was implemented. Generally, healthy food is not for us. The more sugar it has, the better it tastes. Generally, we like what tastes best because taking care of our health is not for us.' (Male, 17 years)

The response pattern among adult FGD participants was complex; while they provided ample evidence of knowledge and comprehension of nutritional information, attitudinal and behaviour change sometimes followed, but not always. Specifically, adults do not necessarily formulate or carry out strategies to change patterns of purchase and consumption of processed foods because they value established preferences and feel that individual acts of consumption will not affect their health.

Adult reponses formulated in response to the information presented on TL labels fall into five strategies. First, some participants stated that they have reduced consumption of products that have high concentrations of fat, sugar or salt. For example, they may consume only a portion of the contents of a package of snacks:

'It might be that I see that there is a lot of fat, so I eat less, and when I see that there is a lot of salt, I shouldn't consume much because I have a bad kidney. So I consume less.' (Female, 57 years)

Second, some participants compensate for the consumption of foods they recognize as unhealthy by accompanying them with other products that have lower concentrations of fat, sugar or salt. For example, they may drink bottled water instead of sweetened beverages at meals that include foods thought to be unhealthy. Similarly, they may compensate by decreasing the frequency of consumption of products with high concentrations of fat, sugar or salt. As one male adult commented regarding sweetened beverages, 'I only drink it sometimes, so it won't do me much harm.' 
A third strategy centres on replacing processed foods that have TL labels with red bars with similar products with medium or low concentrations of fat, sugar or salt. An example is the substitution of soft drinks that have artificial sweeteners instead of sugar:

'You can use [artificial sweeteners]; they are much safer than refined sugar.' (Male, 43 years)

In this context, some participants reported that they use the TL label to guide the purchase and consumption of processed foods because of health problems such as diabetes or hypertension. Generally, these participants know that they should avoid foods with high concentrations of sugar or salt, respectively:

'I use the traffic light food labelling now because of my illness. I see that something is high in fats, high in salt, so I don't buy it and I buy what is low. And it's helped because now my health is quite good.' (Female, 64 years)

Fourth, some participants explained that they have stopped consuming certain products that have high concentrations of fat, sugar or salt:

'That's because we pay attention to what products contain. At least in my case I do and in my family, because products that have a label that says high in salt, sugar or in fat, now we try to avoid them.'

(Female, 60 years)

Among participants who stated that they no longer consume processed foods, older adults were especially vocal in expressing health concerns, especially since they feel that those products are unhealthy compared with unprocessed foods they were familiar with in the past.

Fifth, some FGD participants stated that they have not changed their consumption of processed foods and they continue to consume products with high concentrations of fat, sugar or salt. Adult males in particular explained that they have formed habits that they cannot or will not change or that they believe will not affect their health status in the long run. In contrast, adolescents and young adults reported that price and accessibility are important because school cafeterias offer snacks and beverages with high concentrations of fat, salt and sugar. Also, as in the case of tobacco, young people tend to discount potential future health problems in favour of present satisfaction of perceived need.

\section{Differences by subgroup}

In sum, these findings suggest that FGD participants in different age groups displayed knowledge and comprehension of the information conveyed by the TL label; some developed attitudes that facilitated behaviour change, but others did not. In the latter case, some adults stated that they understand the information presented in the TL label but at the moment of purchase and consumption, preferences related to taste, brand and other factors played a greater role in guiding their behaviour. In short, they may ignore prominently displayed and easily understood information:

'Sometimes we pay no attention to the traffic light label and go more for the junk food than the traffic light.' (Male, 35 years)

The analysis of responses by sex of the FGD participants suggests that there were no differences among males and females in the younger age categories, but that among adults, men and women address issues related to healthy diet differently. Men expressed themselves in theoretical terms rather than through specific behaviours and they were less likely to have changed consumption patterns. In contrast, women viewed themselves as responsible for the purchase and preparation of meals; they reported having formed positive attitudes toward foods that are low in fat, sugar and salt and also that changed attitudes shape their food choices.

Knowledge, comprehension, attitudes and practices related to the TL label also varied by place of residence but not by region. Residents of small and medium-sized towns more often reported buying fresh and unprocessed products in outdoor markets, and many retain contact with the land and still grow their own food. On the other hand, they purchase some of their food in small shops that offer few healthy alternatives. In contrast, consumers in larger cities have little contact with the land but often consume foods purchased in supermarkets, which offer more alternatives to processed foods.

\section{Key informant interviews}

The results of KII are consistent with points raised in the FGD in some respects, but differ in others. Representatives of companies that produce processed foods generally disagreed with the policy that requires the use of the TL label. In particular, they believe that their customers do not find the information useful and that the policy is an unnecessary imposition of the government and multilateral agencies. In contrast, they stated that the GDA label is more useful. Small- and medium-scale manufacturers of processed foods expressed frustration with difficulties in complying with established regulations and stated that sales of some of their products have declined as a consequence. Nevertheless, they accept the policy and expressed their intention to comply with the regulations.

Another perception among KII participants is that the label unfairly demonizes products that have high concentrations of fat, sugar or salt because consumers are given the impression that these products are harmful to their health regardless of the amounts they normally consume. In particular, KII participants believe that consumers interpret red bars on labels as a warning that those products should be avoided entirely. In this regard, they 
stated that the label does not distinguish between products that have concentrations that are slightly above the cut-off points from those that have considerably higher levels. Moreover, they stated that the use of standardized consumption levels (100 g or $100 \mathrm{ml}$ ) does not reflect usual consumption habits and that the amounts of fat, sugar and salt are inflated, particularly when the TL label does not distinguish between naturally occurring and added ingredients.

Another perception expressed by the key informants is that the TL label does little to address the underlying problem of overweight and obesity. They argued that processed foods represent a small proportion of the Ecuadorian diet and that meals prepared at home or in restaurants contribute much more to the problem. Nevertheless, they recognized that many customers prefer products with lower concentrations of sugar, fat and salt. Hence, there is an effort to replace red bars on the label with yellow or green by reducing concentrations of those ingredients. Moreover, large-scale manufacturers avoid introducing new products with high concentrations in any of the three categories. KII participants stated that changing the composition of their products poses technical difficulties because manufacturers must simultaneously decrease concentrations of fat, salt and sugar while responding to consumer taste preferences.

Representatives of supermarket chains recognized that they can offer many alternatives for a diverse clientele, and they have increased the offer of products with lower concentrations of fat, sugar and salt. In contrast, neighbourhood shops are so small that it is difficult to modify stocks, and they respond to customer preferences for snacks and sweetened beverages. The principal factors that shape decisions to buy in small shops are brand and price, and consumers spend little time observing nutritional information or comparing alternatives.

\section{Analysis of regulations and structured observations of processed food labels}

Results of FGD and KII were supported by structured observations of the legal and regulatory framework and of TL labels on processed food packages. Key informants' perceptions that implementation of the policy is complicated because of inconsistencies in the regulations are supported by the fact that two parallel regulations were published on the same day in 2014 by the Ministries of Public Health and Production, respectively. Not only are the two regulations inconsistent in some regards, but also they both differ from previously established technical requirements. TL labels vary considerably in size and location, while additional information (including GDA labels) is often confusing and sometimes contradictory. In addition, many products do not carry the label when they should, while others carry the label even though they are not processed.

\section{Discussion}

In summary, we found that the Ecuadorian TL label provides consumers with readily understood information about the content of sugar, salt and fat in processed foods. The label was understood and favourably regarded by FGD participants and it raised awareness of the importance of fat, sugar and salt in the diet. Moreover, the findings suggest that some segments of the population have changed attitudes and have modified patterns of purchase and consumption of processed foods with high concentrations of fat, sugar or salt.

Based on our findings, we conclude that the TL label is an effective mechanism for comunicating information about the fat, sugar and salt in processed food. Its simple graphical display uses a familiar and easily interpreted colour scheme and text that contributed to FGD participants' knowledge and comprehension that, in some cases, modified attitudes and practices related to the purchase and consumption of processed foods. Nevertheless, while the TL label can contribute to the ability to make healthy choices, it does not necessarily lead to changes in consumption patterns, so it is important to understand the decision-making process in different population groups with respect to processed foods ${ }^{(27-29)}$.

Several factors have limited the potential effectiveness of the Ecuadorian TL label. First, implementation has not been accompanied by effective promotion activities, which would optimize active consumer participation in the process. Similarly, appropriate monitoring and evaluation components would ensure continuity and compliance with the policy. Together, these components could contribute to a convergence of priorities among consumers, the food and beverage industry, and health professionals ${ }^{(29)}$.

Second, the label should be located on the front of packages in order to enhance visibility and potential impact $^{(16,27,28)}$.

Third, as is the case elsewhere, the food and beverage industry has used its political and economic power to influence nutrition policy, having promoted the elimination or modification of the TL label ${ }^{(10)}$. It is incumbent upon the Ecuadorian Government to maintain the label despite pressures; other measures, such as the recent imposition of a tax on sweetened beverages, are also appropriate responses to the coming storm of overweight, obesity and chronic disease. Other countries should adopt a TL label; promote healthy choices regarding foods that contain added sugar, salt or fat; and reach out to food and beverage industries to motivate modifications in the content of processed foods.

\section{Strengths and limitiations}

The present study has a particular set of strengths and limitations. Qualitative research techniques were employed because they provide an understanding of perceptions and opinions that shape individual and collective behaviour. 
Through the use of interrelated field-based methods, data were collected, organized, validated and analysed in order to allow for the emergence of new insights and theory ${ }^{(21,24)}$. The research design allowed for inclusion of critical dimensions of age, sex and geographical distribution in order to take into account potential patterns of similarities and differences. As is true for qualitative research in general, the selection of FGD and KII participants was not based on a representative sampling scheme, so it is not possible to extrapolate the results in a statistical sense. Rather, the findings are based on a theoretical sampling strategy, through which participants were selected for inclusion in the study based on their ability to contribute to an evolving understanding of perceptions and evidence of knowledge, comprehension, attitudes and practices $^{(24,30-32)}$. Hence, these findings support the inclusion and continuation of the TL label policy in Ecuador and should be of use in devising similar policies and strategies elsewhere in Latin America and the world.

\section{Acknowledgements}

Acknowledgements: The authors extend their thanks to the Ecuadorian Ministry of Health and to UNICEF and the Pan American Health Organization (PAHO)/WHO, which supported this study. They are grateful to Mercedes Katherine Silva of UNICEF and to Miguel Malo (former Vice Minister of Health), Manuel Peña and Enrique Jacoby of PAHO, who supported the Ministry of Public Health's initiative to develop the nutritional label. The Universidad San Francisco de Quito's Center for Technological Transfer and Development (Victor Viteri, Lenin Vinueza, Julio Pillajo and Yadira Mamallacta) provided administrative support. Local counterparts Amaya Carrasco, Anabel Ponce, Elizabeth Loor, Patricia Moreno and Diana Chavez facilitated fieldwork. Neidy Marten and Juan Aguaguil provided transcription services. The authors are grateful to the focus group participants and key informants. Finally, the authors are grateful for the helpful comments of three anonymous reviewers. Financial support: Support was provided by the Ecuador offices of UNICEF and the PAHO. UNICEF and PAHO had no role in the design, analysis or writing of this article. Conflict of interest: None. Authorship: W.B.F. was the principal investigator and responsible for overall project design, data analysis and manuscript preparation. W.F.W. was responsible for research design and implementation, conducting the KII, data analysis and manuscript preparation. G.R.-M. directed and conducted the FGD and structured observations, and contributed to data analysis and manuscript preparation. T.N. prepared the literature review and contributed to manuscript preparation. P.R. carried out field research and contributed to data analysis. Ethics of buman subject participation: This study was conducted according to the guidelines laid down in the Declaration of Helsinki and all procedures involving human subjects/patients were approved by the Bioethics Committee of the Universidad San Francisco de Quito. Written informed consent was obtained from FGD participants and verbal consent was obtained from KII participants. Verbal consent was witnessed and formally recorded.

\section{Supplementary material}

To view supplementary material for this article, please visit http://dx.doi.org/10.1017/S1368980016002457

\section{References}

1. Freire WB, Ramírez-Luzuriaga MJ, Belmont P et al. (2014) Tomo I: Encuesta Nacional de Salud y Nutrición ENSANUTECU 2012 (Volume I: National Health and Nutrition Survey ENSANUT-ECU 2012). Quito: Ministerio de Salud Pública/ Instituto Nacional de Estadística y Censos.

2. NCD Risk Factor Collaboration (2016) Trends in adult bodymass index in 200 countries from 1975 to 2014: a pooled analysis of 1698 population-based measurement studies with 19.2 million participants. Lancet 387, 1377-1396.

3. Krug EG (2016) Trends in diabetes: sounding the alarm. Lancet 387, 1485-1486.

4. Chan M (2013) WHO Director-General addresses health promotion conference. http://www.who.int/dg/speeches/ 2013/health_promotion_20130610/en/ (accessed May 2016).

5. Kelly B, Hughes C, Chapman K et al. (2009) Consumer testing of the acceptability and effectiveness of front-of-pack food labelling systems for the Australian grocery market. Health Promot Int 24, 120-129.

6. Food Standards Agency (2006) Full regulatory impact assessment. http://tna.europarchive.org/20130129064400/ http://www.food.gov.uk/multimedia/pdfs/signpostingria. pdf (accessed August 2016).

7. Draper AK, Adamson AJ, Clegg S et al. (2013) Front-of-pack nutrition labelling: are multiple formats a problem for consumers? Eur J Public Health 23, 517-521.

8. Vasiljevic M, Pechey R \& Marteau TM (2015) Making food labels social: the impact of colour of nutritional labels and injunctive norms on perceptions and choice of snack foods. Appetite 91, 56-63.

9. Van Kleef E, Van Trijp H, Paeps F et al. (2008) Consumer preferences for front-of-pack calories labelling. Public Health Nutr 11, 203-213.

10. Mandle J, Tugendhaft A, Michalow J et al. (2015) Nutrition labelling: a review of research on consumer and industry response in the global South. Glob Health Action 8; available at http://dx.doi.org/10.3402/gha.v8.25912

11. Campos S, Doxey J \& Hammond D (2011) Nutrition labels on pre-packaged foods: a systematic review. Public Health Nutr 14, 1496-1506.

12. Gonzalez-Zapata LI, Alvarez-Dardet C, Ortiz-Moncada R et al. (2009) Policy options for obesity in Europe: a comparison of public health specialists with other stakeholders. Public Health Nutr 12, 896-908.

13. Gracia A, Loureiro ML \& Nayga RM (2009) Consumers' valuation of nutritional information: a choice experiment study. Food Qual Prefer 20, 463-471.

14. Van Herpen E, Seiss E \& van Trijp HC (2012) The role of familiarity in front-of-pack label evaluation and use: a comparison between the United Kingdom and The Netherlands. Food Qual Prefer 26, 22-34.

15. Hersey JC, Wohlgenant KC, Arsenault JE et al. (2013) Effects of front-of-package and shelf nutrition labeling systems on consumers. Nutr Rev 71, 1-14. 
16. Hawley KL, Roberto CA, Bragg MA et al. (2013) The science of front-of-package labels. Public Health Nutr 16, 430-439.

17. Borgmeier I \& Westenhoefer J (2009) Impact of different food label formats on healthiness evaluation and food choice of consumers: a randomized-controlled study. BMC Public Health 9, 184

18. Ecuador (2014) Expedíse el reglamento sanitario sustiutivo de etiquetado de alimentos procesados para el consumo humano (Publish the substitute sanitary regluation for labelling of processed foods for human consumption). Registro Oficial II, 1-8.

19. Food Standards Agency (2013) Front of pack nutrition labelling: joint response to consultation. http://www.food.gov.uk/ sites/default/files/multimedia/pdfs/consultationresponse/ frontofpacklabelling-response.pdf (accessed May 2016).

20. Food Standards Agency (2013) Guide to creating a front of pack (FoP) nutrition label for pre-packed products sold through retail outlets. https://www.gov.uk/government/ uploads/system/uploads/attachment_data/file/300886/ 2902158_FoP_Nutrition_2014.pdf (accessed May 2016).

21. Patton MQ (2002) Qualitative Research and Evaluation Methods, 3rd ed. Thousand Oaks, CA: SAGE Publications, Inc.

22. Ulin PR, Robinson ET \& Tolley EE (2005) Qualitative Methods in Public Health: A Field Guide for Applied Research. San Francisco, CA: Jossey-Bass.

23. Krueger RA \& Casey MA (2000) Focus Groups: A Practical Guide for Applied Research, 3rd ed. Thousand Oaks, CA: SAGE Publications, Inc.

24. Strauss A \& Corbin J (1998) Basics of Qualitative Research: Procedures and Techniques for Developing Grounded
Theory, 2nd ed. Thousand Oaks, CA: SAGE Publications, Inc.

25. Peña M, Montoya R \& Rodríguez M (2014) La publicidad de productos comestibles en la TV-Ecuador (Advertising of edible products on TV-Ecuador). Rev Inform OPS/OMS (Inform J PAHO/WHO) 32, 43-46.

26. Andreyeva T, Kelly IR \& Harris JL (2011) Exposure to food advertising on television: associations with children's fast food and soft drink consumption and obesity. Econ Hum Biol 8, 221-233.

27. Sacks G, Rayner M \& Swinburn B (2009) Impact of front-of-pack 'traffic-light' nutrition labelling on consumer food purchases in the UK. Health Promot Int 24, 344-352.

28. Van Kleef E \& Dabevos H (2015) The growing role of frontof-pack nutrition profile labeling: a consumer perspective on key issues and controversies. Crit Rev Food Sci Technol 55, 291-303.

29. Koen N, Blaauw R \& Wentzel-Viljoen E (2016) Food and nutrition labelling: the past, present and the way forward. $S$ Afr J Clin Nutr 29, 13-21.

30. Creswell JW (1998) Qualitative Inquiry and Research Design: Choosing Among Five Traditions. Thousand Oaks, CA: SAGE Publications, Inc.

31. Glaser B \& Strauss A (1967) Discovery of Grounded Theory. Chicago, IL: Aldine.

32. Gobo G (2004) Sampling, representativeness and generalizability. In Qualitative Research Practice, pp. 435-456 [C Seale, G Gobo, JF Gubrium et al., editors]. London: SAGE Publications Ltd. 